\title{
Illumination Uniformity Evaluation on Visible Light and Near-Infrared Face Images
}

\author{
Haidi Ibrahim, Senior Member, IACSIT, and Kuo Chue Neo
}

\begin{abstract}
In this paper, illumination uniformity on face images has been accessed. A database of 1056 visible light face images and 1056 near-infrared face images has been used in our assessment process. This database uses 22 volunteers, irrespective to race and gender. Each volunteer had to attend two photography sessions. Our investigation on intensity distributions, and intensity differences, supports the theory that near-infrared face images are more robust towards illumination variations.
\end{abstract}

Index Terms-Digital image processing, face images, face detection, illumination inhomogeneity, illumination uniformity, near-infrared face images, visible light face images.

\section{INTRODUCTION}

Security is becoming one of the crucial aspects in our life nowadays. Many surveillance and security systems have been introduced, including biometric systems. Biometric systems use some unique features on human being, such as face, iris, fingerprint, or voice, to detect, identify or verify a person. One of popular biometric features used these days is the face. Biometric systems based on face are easily accepted by society. This is mostly due to the acquisition of face features is normally involving nonintrusive methods [1].

Face images can be obtained within the range of visible light, or infrared spectrum. The wavelength for visible light is in between $0.4 \mu \mathrm{m}$ to $0.7 \mu \mathrm{m}$. The spectrum of infrared is much wider, which is from $0.7 \mu \mathrm{m}$ to $14.0 \mu \mathrm{m}$. Some of the useful infrared bands that are used in face recognition systems are:

$$
\begin{aligned}
& \text { - } 0.7 \mu \mathrm{m} \text { to } 0.9 \mu \mathrm{m} \quad \text { : near-infrared } \\
& \text { - } 0.9 \mu \mathrm{m} \text { to } 2.4 \mu \mathrm{m} \quad \text { : short-wave infrared } \\
& \text { - } 3.0 \mu \mathrm{m} \text { to } 5.0 \mu \mathrm{m} \text { : mid-wave infrared } \\
& \text { - } 8.0 \mu \mathrm{m} \text { to } 14.0 \mu \mathrm{m} \text { : long-range infrared }
\end{aligned}
$$

Near-infrared and short-wave infrared are related to the reflected solar radiation. On the other hand, the mid-wave infrared and the long-range infrared, deal with the heat emitted by the objects [2].

One of the most challenging problems for biometric systems based on face images is shadow. Shadows are commonly resulted from improper lighting conditions, where the light sources are distributed unevenly around the face.

Manuscript received October 14, 2012; revised November 15, 2012. This work was supported in part by the Universiti Sains Malaysia's Short Term Research Grant with account number 304/PELECT/60311013.

The authors are with the School of Electical \& Electronic Engineering Engineering Campus, Universiti Sains Malaysia, 14300 Nibong Tebal, Penang, Malaysia (e-mail: haidi_ibrahim@ ieee.org, nkc99131@ student.usm.my).
Shadow can significantly change the appearance of human face in the acquired images, and as a consequence, can significantly affect the results of the recognition process. Therefore, many researchers are now trying to solve the shadow related problem in face images, for the use in biometric system [3].

In this paper, we investigate some properties of facial images acquired under visible light, and compare it with the corresponding properties of near-infrared face images. Our aim is to inspect the illumination uniformity in these two image types. The method used in this paper, and the results obtained are presented in the next section. The last section of this paper will summarize our finding.

\section{Method, Results And Discussions}

A face database, known as the Active Near-Infrared Differential Imaging (ANIDI), which has been provided by the Center for Vision, Speech and Signal Processing (CVSSP), University of Surrey, United Kingdom, was downloaded from [4]. This database consists of a set of visible light face images of 23 volunteers (i.e. 1080 photos), and a corresponding set of near-infrared face images (i.e. 1080 photos). In addition to face images, coordinates of eyes in each image are also provided.

Fig. 1 shows face images obtained from one volunteer. Fig. 1 (a) to (i) are visible light face images. Fig. 1(a) to (d) were taken during the first photography session, while Fig. 1 (f) to (i) were taken a few weeks after the first session. The lighting conditions are as follows:

- Lighting 1 and 6: Illuminate the faces from the left

- Lighting 2 and 7: Illuminate the faces from the bottom

- Lighting 3 and 8: Illuminate the faces from the right

- Lighting 4 and 9: Illuminate the faces from the top

In addition to the visible light illumination sources, at each lighting condition, the volunteers were also been photographed by near-infrared sources. The corresponding near-infrared images, which have been deduced from Fig. 1 (a) to (i), are shown by Fig. 1 (j) to (q). The process of obtaining these images is well explained in [5] and [6].

In our work, although there are 23 volunteers in the database, we only used images from 22 volunteers. This is because the sample from volunteer with identification number 042 is incomplete. Therefore, in our work, we only used 1056 visible light face images, and 1056 near-infrared face images. 


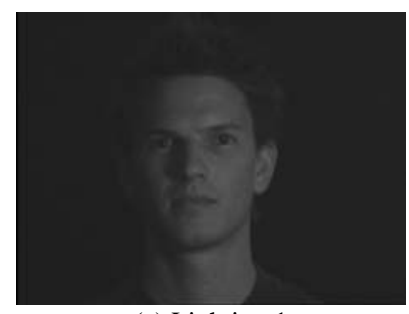

(a) Lighting 1

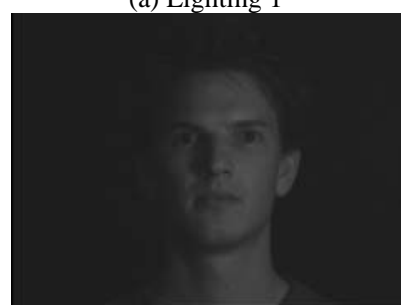

(f) Lighting 6

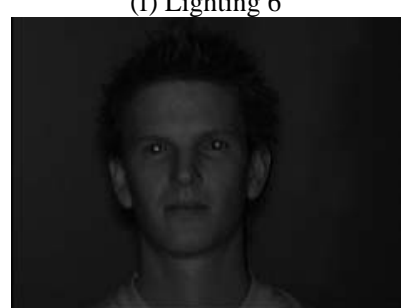

(j) Lighting 1

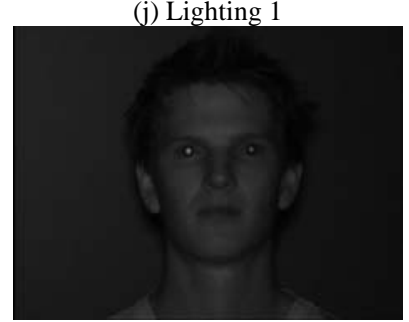

(n) Lighting 6

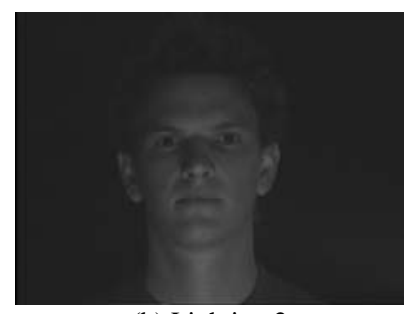

(b) Lighting 2

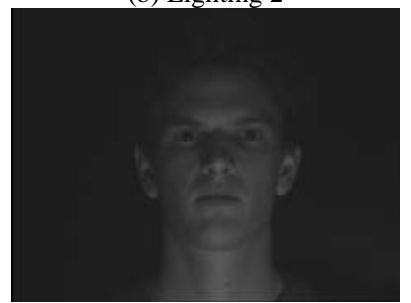

(g) Lighting 7

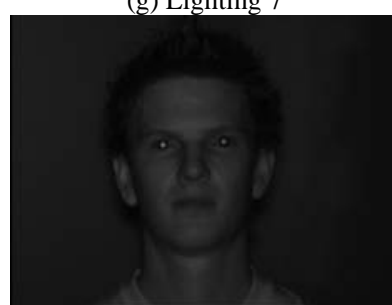

(k) Lighting 2

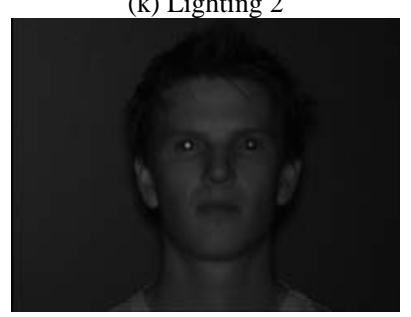

(o) Lighting 7

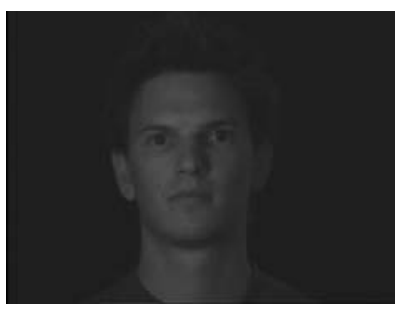

(c) Lighting 3

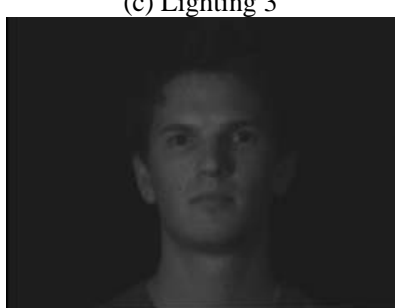

(h) Lighting 8

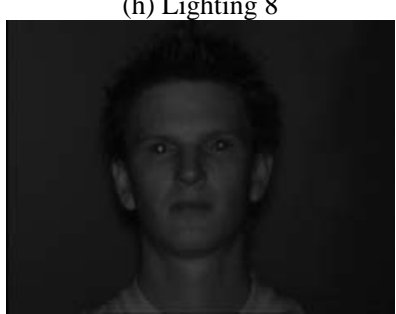

(l) Lighting 3

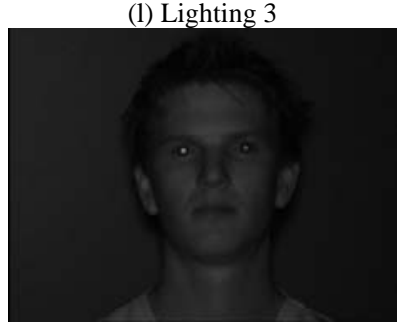

(p) Lighting 8

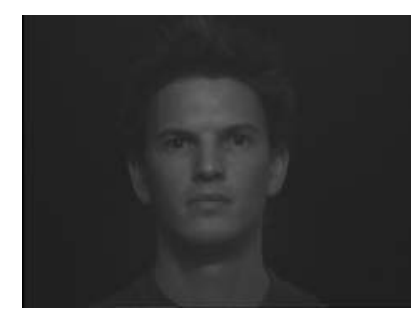

(d) Lighting 4

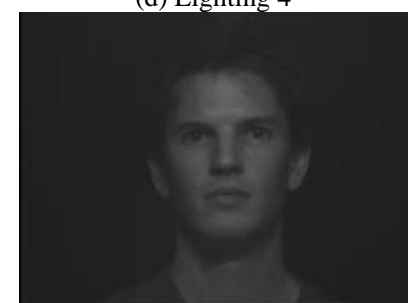

(i) Lighting 9

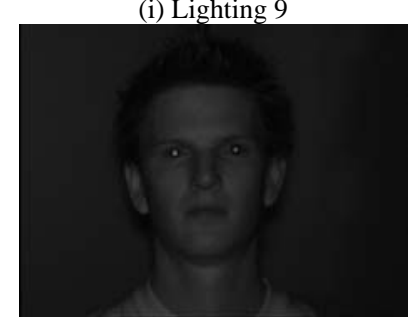

(m) Lighting 4

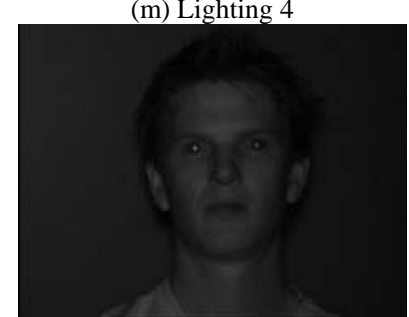

(q) Lighting 9

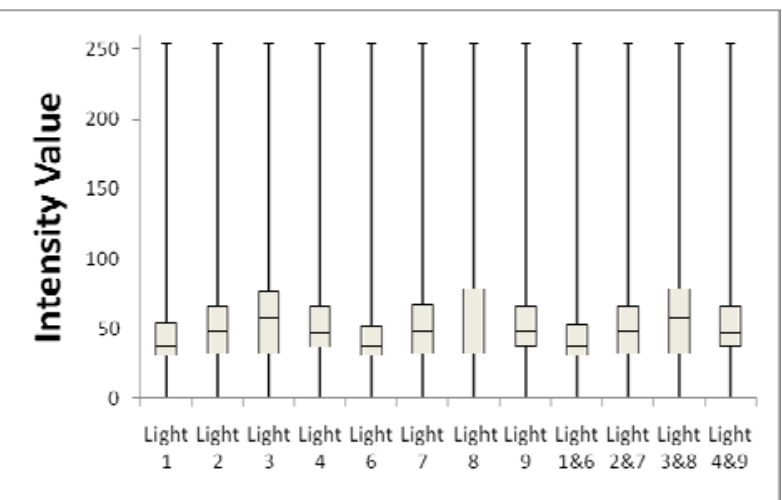

(a) Visible light face images

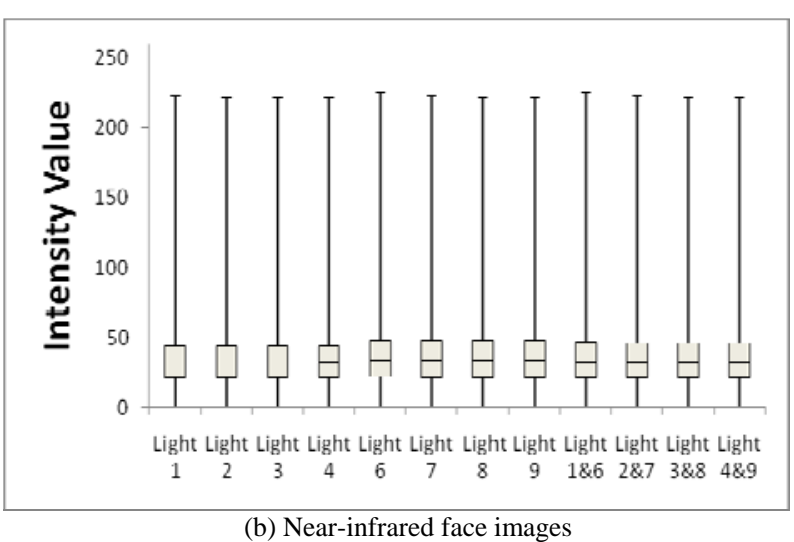

Fig. 2. Intensity distributions for each lighting condition.
The box-and-whiskers plots of the intensity distribution for each lighting condition are presented in Fig. 2. In this plot, in addition to individual lighting condition, we also combine the similar lighting condition together. As shown by Fig. 2(a), under visible light illuminations, face images occupy the full intensity range, which is from 0 to 255 . On the other hand, the maximum intensity value for the near-infrared face images is less than 250. This figure shows that for both visible light and near infrared face images, the distributions are dominated by low intensity values. That is the reason why in Fig. 1, the images are dark. However, Fig. 2(b) shows an interesting fact, that is, regardless the lighting condition, the intensity distributions in near-infrared face images are nearly unchanged. The locations of the median value are also nicely positioned nearly to the middle between the first and third quartiles.

By using the information regarding to locations of the eyes in each image, we came out with one simple quality measure, in order to measure the illumination uniformity on the face. By using the column location of the left eye $E_{L}$ and the column location of the right eye $E_{R}$, the distance between two eyes, $d$, can be estimated:

$$
d=E_{R^{-}} E_{L}
$$

The middle location, $m$, between two eyes can be determined as:

$$
m=\left(E_{R}+E_{L}\right) / 2
$$


In order to estimate the uniformity, four simple assumptions have been used:

- The head is in frontal position

- $\quad$ The head is horizontally symmetry, by taking $m$ as the reflective axis

- The intensity distribution on the left side is same with the intensity distribution on the right side, if the illumination is uniform.

- The width of the head is equal to $2 d$.

Therefore, the input image has been cropped to fit the above assumptions. The left border, $L$, and the right border, $R$, are calculated as:

$$
\begin{gathered}
L=m-d \\
R=m+d
\end{gathered}
$$

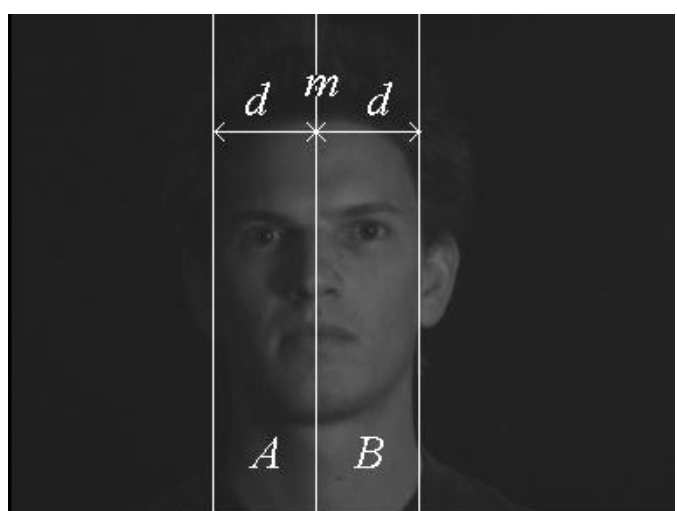

Fig. 3. The face image has been cropped and divided into two sections; $A$

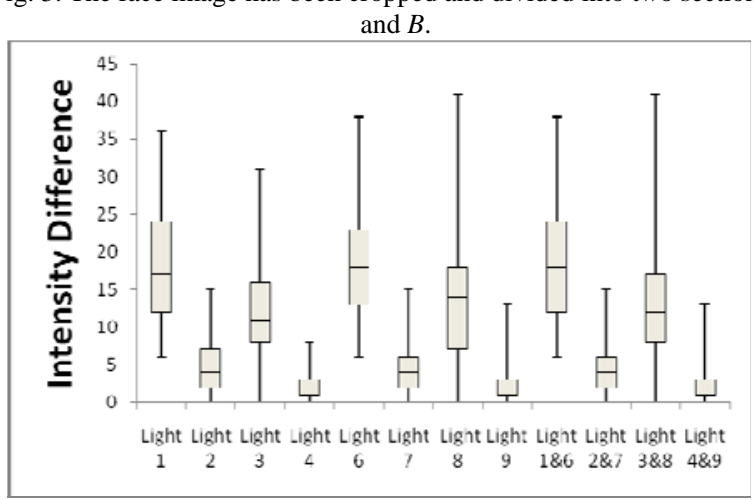

(a) Visible light face images.

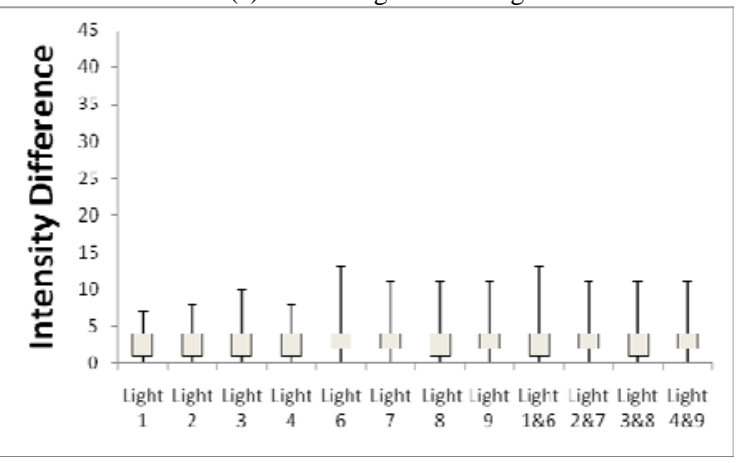

(b) Near-infrared face images.

Fig. 4. Distributions of intensity difference, between region A and B (see Fig. 3)
This image partitioning is shown in Fig. 3. The face image is divided into two sections, i.e. section $A$, and section $B$. If $a$ is the average intensity value inside region $A$, and $b$ is the average intensity value inside region $B$, the intensity difference, which we use to measure the illumination uniformity, is defined as:

$$
\text { Intensity Difference }=|a-b|
$$

Uniform illumination will produce small intensity difference, because under this condition, the distribution of intensity values in region $A$ should be the same as the one in region $B$.

As shown in Fig. 4, the intensity differences of the visible light face images are relatively higher as compared with the intensity differences of the near-infrared face images. For the near-infrared face images, the intensity differences are very small, where all of the third quartiles are equal to four. For the visible light face images, the intensity differences are dependent to the lighting condition. Fig. 4(a) suggests that among the four lighting condition tested, the illumination problem is less crucial when the faces were illuminated from the top, as indicated by low intensity differences. This is proven when we inspect Fig. 1. Not much shadow can be seen from this lighting condition.

\section{SUMMARY}

We investigated the intensity distribution properties of the dataset. From these works, we prove that the near-infrared face database is more robust towards different lighting conditions, compared with the visible light face database. The work presented here also can be used as a guidance to produce a useful image quality measure, or to detect any illumination inhomogeneity on the face images.

\section{REFERENCES}

[1] A. K. Jain, A. Ross, and S. Prabhakar, "An introduction to biometric recognition," IEEE Transactions on Circuits and Systems for Video Technology, vol. 14, no. 1, pp. 4-20, 2004.

[2] S. G. Kong, J. Heo, B. R. Abidi, J. Paik, and M. A. Abidi, "Recent advances in visual and infrared face recognition - a review," Computer Vision and Image Understanding, vol. 97, pp. 103-135, 2005.

[3] H. Wang, S. Z. Li, and Y. Wang, "Face recognition under varying lighting conditions using self quotient image," in Proceedings of the Sixth IEEE International Conference on Automatic Face and Gesture Recognition, pp. 819-824, 2004.

[4] Centre for Vision. Speech and Signal Processing, University of Surrey. Near-infrared and visible light face database. [online] http://www.ee.surrey.ac.uk/CVSSP/Datasets/NIRVis

[5] X. Zou, J. Kittler and K. Messer, "Ambient illumination variation removal by active near-IR imaging," in Proceedings of IAPR International Conference on Biometric, pp. 19-25, 2006.

[6] X. Zhou, J. Kittler and K. Messer, "Face recognition using active near-IR illumination," in Proceedings of British Machine Vision Conference, pp. 209-219, 2005. 